\title{
No aggravation of congenital thrombotic thrombocytopenic purpura by mRNA-based vaccines against COVID-19: a Japanese registry survey
}

\author{
Eriko Hamada ${ }^{1} \cdot$ Kazuya Sakai $^{1} \cdot$ Shinya Yamada ${ }^{1} \cdot$ Masayuki Kubo $^{1} \cdot$ Masaki Hayakawa ${ }^{1} \cdot$ Masanori Matsumoto $^{1}$ (i)
}

Received: 13 January 2022 / Accepted: 26 January 2022 / Published online: 4 February 2022

(c) The Author(s), under exclusive licence to Springer-Verlag GmbH Germany, part of Springer Nature 2022

\section{Dear Editor:}

Congenital thrombotic thrombocytopenic purpura (cTTP) is an ultra-rare disorder caused by severe ADAMTS13 deficiency [1]. Between 2001 and 2020, only 226 patients with cTTP were reported worldwide [2]. As of 2021, we identified 67 patients with cTTP in Japan alone [3, 4]. Theoretically, patients with cTTP are at a high risk for thrombosis due to COVID-19 infection. This is attributed to the increase in plasma von Willebrand factor levels and decrease in plasma ADAMTS13 activity with disease severity in patients with COVID-19 infection [5].

Recently, Dykes et al. reported a case in which COVID19 vaccination exacerbated the cTTP condition [6]. This patient developed an acute ischemic stroke with mild thrombocytopenia shortly after receiving the COVID-19 vaccine (mRNA-1273). To date, over 10 cases of de novo immune TTP have been reported following COVID-19 vaccination worldwide $[7,8]$. However, there have been no other reports of COVID-19 vaccine-induced flare of cTTP. In Japan, two types of mRNA-based vaccines, BNT162b2 (Pfizer-BioNTech) and mRNA-1273 (Moderna), are available. We administered a questionnaire survey in Japanese patients with cTTP regarding the adverse events after vaccination in October 2021, when double doses of vaccinations were almost completed in Japanese applicants who were $\geq 12$ years of age.

The questionnaire was sent to physicians who were treating patients with cTTP. Of the 67 Japanese patients with cTTP, 58 were alive and 6 were under 12 years of age. According to the questionnaire survey of 42 patients, 38 had completed vaccination twice, and 4 patients had not

Masanori Matsumoto

mmatsumo@naramed-u.ac.jp

1 Department of Blood Transfusion Medicine, Nara Medical University, Shijyo-cho 840, Kashihara, Nara 634-8522, Japan yet received them. Table 1 presents the results for these 38 patients. Of these, 24 patients were administered prophylactic fresh frozen plasma (FFP) infusions at 2-3 weeks interval to prevent TTP episodes, and three were involved in clinical trials of recombinant ADAMTS13. Regarding the type of COVID-19 vaccination, 35 patients received BNT162b2, one received mRNA-1273, and the data for two patients were unknown. No severe adverse effects of vaccination, especially exacerbation of cTTP, were observed, and platelet counts after vaccinations were unchanged compared with the pre-vaccination levels. All patients receiving FFP infusion were vaccinated within 2 weeks of FFP infusion. In contrast, the patient reported by Dykes et al. underwent vaccination 6 weeks after OctaPlas ${ }^{\mathrm{TM}}$ (solvent/detergent treated, pooled human plasma) infusion and developed ischemic stroke 1 week after vaccination [6]. We hypothesize that a long interval between plasma infusion and vaccination could be a risk factor for cTTP exacerbation.

We believe that all patients with cTTP should receive COVID-19 vaccination based on the following considerations:

1) It is desirable to maintain a platelet count of $50,000 / \mu \mathrm{L}$ or higher at the time of vaccination. This is due to the possibility of intramuscular hematoma in cases of severe thrombocytopenia.

2) Patients who have received prophylactic FFP infusion should be vaccinated as soon as possible (preferable within 7 days) after the infusion.

3) Patients who have not received regular FFP infusion may undergo vaccination during stable conditions.

Physicians should pay attention to any changes in the physical condition of patients after vaccination. 
Table 1 Characteristics of 38 patients who received COVID19 vaccines

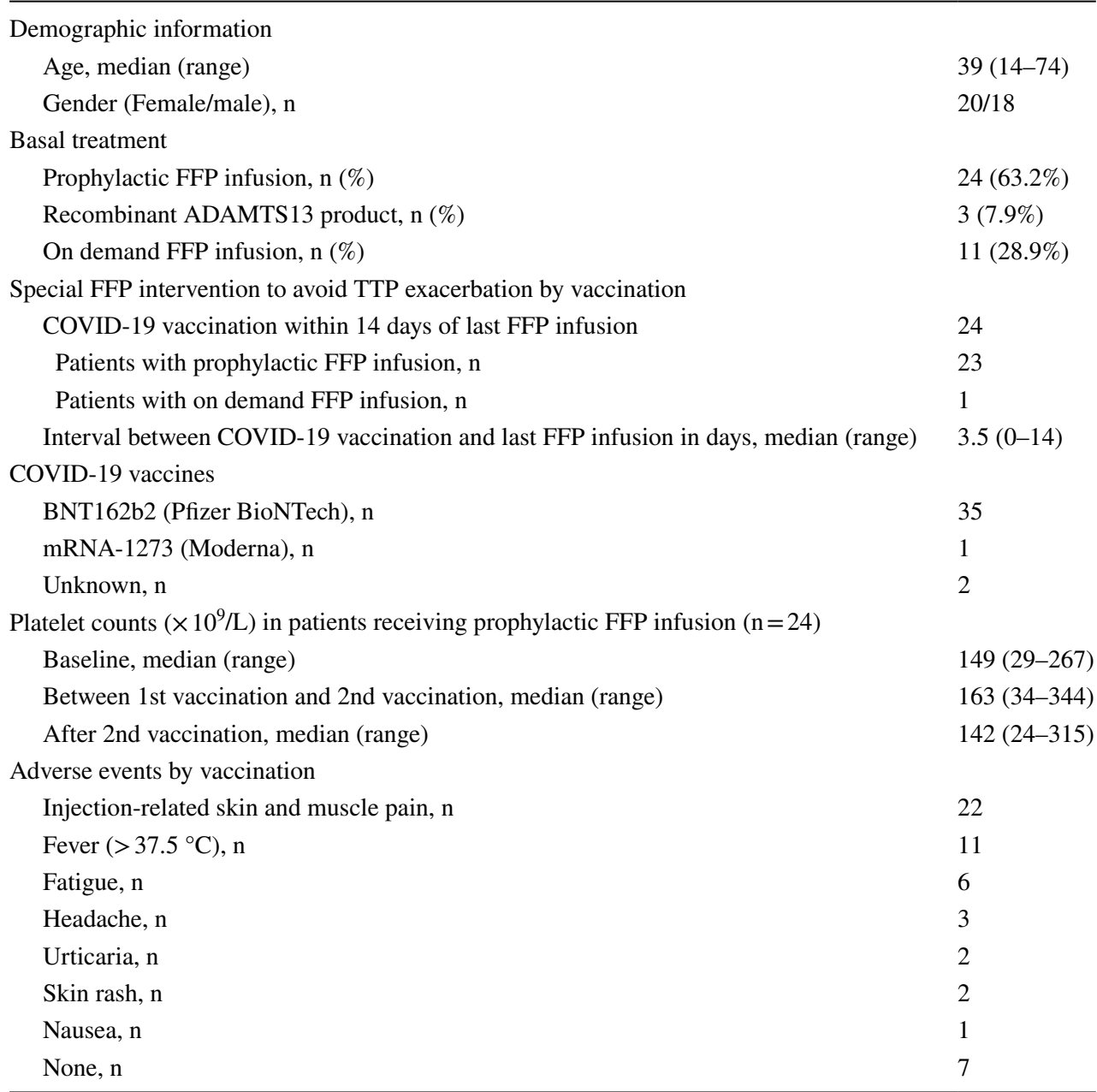

$F F P$, fresh frozen plasma
Acknowledgements The authors thank the physicians for sharing the data of patients with cTTP.

Funding This study was supported by research grants from the Ministry of Health, Labour, and Welfare of Japan.

\section{Declarations}

Ethical approval and consent to participate This study was approved by the ethics committee of Nara Medical University and was compliant with the Declaration of Helsinki. Informed consent was obtained from all patients included in this study.

Conflict of interest M. Matsumoto is a member of the clinical advisory bord of Takeda Yakuhin. He is also an inventor of ADAMTS13 act-ELISA. The remaining authors declare no competing financial interests.

\section{References}

1. Kremer Hovinga JA, George JN (2019) Hereditary thrombotic thrombocytopenic purpura. N Engl J Med 381:1653-1662. https:// doi.org/10.1056/NEJMra1813013

2. Borogovac A, Reese JA, Gupta S, George JN (2021) Morbidities and mortality in patients with hereditary thrombotic thrombocytopenic purpura. Blood Adv. https://doi.org/10.1182/bloodadvan ces. 2021005760

3. Fujimura Y, Matsumoto M, Isonishi A, Yagi H, Kokame K, Soejima K, Murata M, Miyata T (2011) Natural history of UpshawSchulman syndrome based on ADAMTS13 gene analysis in Japan. J Thromb Haemost 9(Suppl 1):283-301. https://doi.org/ 10.1111/j.1538-7836.2011.04341.x

4. Sakai K, Fujimura Y, Miyata T, Isonishi A, Kokame K, Matsumoto M (2021) Current prophylactic plasma infusion protocols do not adequately prevent long-term cumulative organ damage in the Japanese congenital thrombotic thrombocytopenic purpura cohort. Br J Haematol 194:444-452. https://doi.org/10.1111/bjh.17560 
5. Mancini I, Baronciani L, Artoni A, Colpani P, Biganzoli M, Cozzi G, Novembrino C, Boscolo Anzoletti M, De Zan V, Pagliari MT, Gualtierotti R, Aliberti S, Panigada M, Grasselli G, Blasi F, Peyvandi $F$ (2021) The ADAMTS13-von Willebrand factor axis in COVID-19 patients. J Thromb Haemost 19:513-521. https://doi. org $/ 10.1111 /$ jth. 15191

6. Dykes KC, Kessler CM (2022) First report of COVID-19 vaccine induced flare of compensated congenital thrombotic thrombocytopenic purpura. Blood Coagul Fibrinolysis 33:71-73. https://doi. org/10.1097/MBC.0000000000001097

7. de Bruijn S, Maes MB, De Waele L, Vanhoorelbeke K, Gadisseur A (2021) First report of a de novo iTTP episode associated with an mRNA-based anti-COVID-19 vaccination. J Thromb Haemost 19:2014-2018. https://doi.org/10.1111/jth.15418

8. Yoshida K, Sakaki A, Matsuyama Y, Mushino T, Matsumoto M, Sonoki T, Tamura S (2021) Acquired thrombotic thrombocytopenic purpura following BNT162b2 mRNA coronavirus disease vaccination in a Japanese patient. Intern Med. https://doi.org/10. 2169/internalmedicine.8568-21

Publisher's note Springer Nature remains neutral with regard to jurisdictional claims in published maps and institutional affiliations. 